\title{
Electron Transport in Modulation-Doped InAlAs/InGaAs/InAlAs Heterostructures in High Electric Fields
}

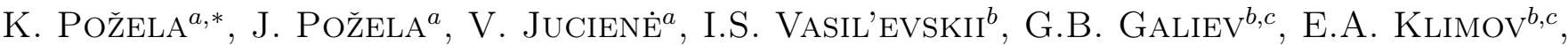 \\ A. SuŽIedélis ${ }^{a}$, N. Žurauskiente ${ }^{a}$, V. StankeviČ ${ }^{a}$, S. Keršulis ${ }^{a}$ And Č. PašKeviČ ${ }^{a}$ \\ ${ }^{a}$ Semiconductor Physics Institute, Center for Physical Sciences and Technology, Goštauto 11, Vilnius, Lithuania \\ ${ }^{b}$ National Research Nuclear University MEPhI, Moscow 115409, Russia \\ ${ }^{c}$ Institute of Microwave Semiconductor Electronics, Russian Academy of Sciences, Moscow 117105, Russia \\ The following peculiarities of electron transport in $\mathrm{In}_{0.53} \mathrm{Ga}_{0.47} \mathrm{As} / \mathrm{In}_{0.52} \mathrm{Al}_{0.48} \mathrm{As}$ quantum wells with \\ $\delta$-Si-doped $\mathrm{In}_{0.52} \mathrm{Al}_{0.48} \mathrm{As}$ barriers at high electric fields are discovered: (1) an enhancement of electron mobility \\ by inserting the InAs phonon wall into the $\mathrm{In}_{0.53} \mathrm{Ga}_{0.47} \mathrm{As} / \mathrm{In}_{0.52} \mathrm{Al}_{0.48} \mathrm{As}$ quantum well, as well as increasing the \\ InAs content in the modulation-doped $\mathrm{In}_{0.8} \mathrm{Ga}_{0.2} \mathrm{As} / \mathrm{In}_{0.7} \mathrm{Al}_{0.3} \mathrm{As}$ heterostructure; (2) a large decrease in electron \\ mobility and a change of electron density with increasing electric field in the range of $1-4 \mathrm{kV} / \mathrm{cm}$; (3) a magnetic \\ field dependence of the threshold electric field for intervalley scattering of electrons; and (4) microwave current \\ oscillations in high electric fields.
}

PACS: 72.20.Ht, 72.10.Di, 73.21.Fg, 73.63.Hs, 73.40.Kp

\section{Introduction}

Modulation-doped heterostructures with high electron mobility have found many applications in microwave and terahertz frequency range [1-5]. In these structures, the spatial separation of free electrons from an impurity-doped layer reduces impurity scattering and enhances electron mobility. However, the spatial separation of impurities and free electrons causes specific changes in electron transport in modulation-doped structures, which are not observed in homogeneous doped field-effect transistor structures [6].

In the paper, the large change in electron density and mobility at relatively low electric fields $(1-3 \mathrm{kV} / \mathrm{cm})$ and magnetic field influence on microwave current instabilities at high electric fields in InAlAs/InGaAs/InAlAs double barrier heterostructures with delta-Si doped InAlAs barriers are considered.

\section{Change in electron density and mobility} in InGaAs QWs at low electric fields

Electron transport in the $\mathrm{In}_{0.53} \mathrm{Ga}_{0.47} \mathrm{As} /$ $\mathrm{In}_{0.52} \mathrm{Al}_{0.48} \mathrm{As}$ heterostructures of five types with a quantum well $(\mathrm{QW})$ width of $16 \mathrm{~nm}$ were investigated. The parameters of the structures are shown in Table. Structures 805 and 796 are with inserted thin (1-2 nm) InAs layers and have higher mobility in comparison with structures 804 and 794 without the InAs inserts. The thin electron transparent InAs insert is assumed to be reflecting for optical phonons and is considered as a phonon barrier or phonon wall.

The highest mobility has structure 29B with the increased InAs component in the $\mathrm{In}_{0.8} \mathrm{Ga}_{0.2} \mathrm{As} /$

\footnotetext{
* corresponding author; e-mail: kpozela@pfi.lt
}

TABLE

Hall mobility and electron density, $\mu_{\mathrm{H}}$ and $n_{\mathrm{sH}}$, and electron density $n_{\mathrm{S}}(0.5)$ obtained from the magnetoresistance measurements in samples with length of 10 and $100 \mu \mathrm{m}$, at electric field $F=0.5 \mathrm{kV} / \mathrm{cm}$.

\begin{tabular}{c|c|c|c|c}
\hline \hline Sample & $\mu_{\mathrm{H}}$ & $n_{\mathrm{sH}}$ & \multicolumn{2}{|c}{$n_{\mathrm{s}}(0.5)\left[10^{12} \mathrm{~cm}^{-2}\right]$} \\
\cline { 4 - 5 } & {$\left[\mathrm{cm}^{2} \mathrm{~V}^{-1} \mathrm{~s}^{-1}\right]$} & {$\left[10^{12} \mathrm{~cm}^{-2}\right]$} & $10 \mu \mathrm{m}$ & $100 \mu \mathrm{m}$ \\
\hline $29 \mathrm{~B}$ & 12300 & 1.44 & 1.5 & 1.5 \\
805 & 8400 & 1.22 & 1.5 & 3.3 \\
796 & 6100 & 2.53 & 2.55 & 4.5 \\
804 & 6200 & 0.98 & 1.4 & 2.0 \\
794 & 5500 & 3.49 & 3.2 & 4.5
\end{tabular}

$\mathrm{In}_{0.7} \mathrm{Al}_{0.3}$ As heterostructures. Figure 1 shows the dependences of current $I$ along the InAlAs/InGaAs/InAlAs QW layer with modulation-doped InAlAs barriers on mean electric field strength $F=U / d$, where $U$ is the applied voltage, in the samples with different source-drain lengths $d=10$ and $100 \mu \mathrm{m}$. The widths of the mesastructure and ohmic contact $(\mathrm{Al} / \mathrm{Ni} / \mathrm{Ge})$ are $100 \mu \mathrm{m}$.

To separate the contributions of changes in electron mobility and electron density to the current dependence on electric fields, the mobility was determined from magnetoresistance measurements in short $(10 \mu \mathrm{m})$ samples. Figure 2 shows the field dependences of the mobility in the investigated structures. One can see that at the fields $F$ of $0-4 \mathrm{kV} / \mathrm{cm}$, the mobility in samples 796 and 805 with the inserted InAs barriers, as well as in the samples with the increased InAs content in the modulation-doped heterostructure are larger than that in the structures without the InAs barriers. At fields $F<0.5 \mathrm{kV} / \mathrm{cm}$, the mobility in sample 29B is 1.7 times larger than in samples 794 and 804 without the barriers. The mobility in sample 805 with two inserted InAs barriers, and in sample 796 with the single InAs barrier is 1.4 and 1.1 times 


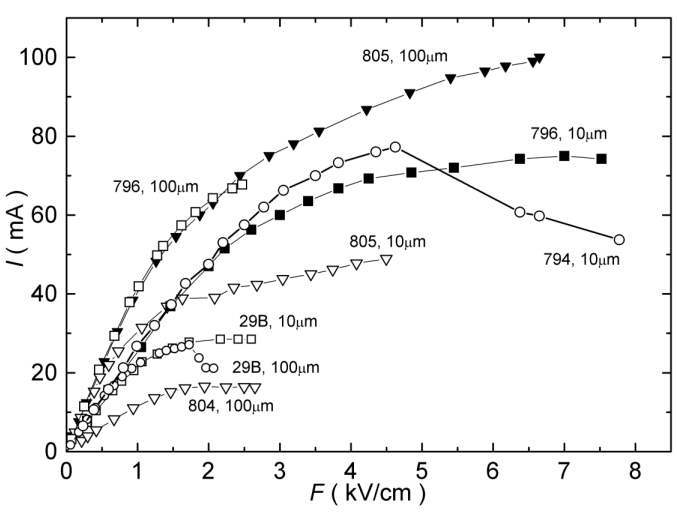

Fig. 1. Field dependences of the current along the InAlAs/InGaAs/InAlAs quantum well for different samples with source-drain lengths of 10 and $100 \mu \mathrm{m}$.

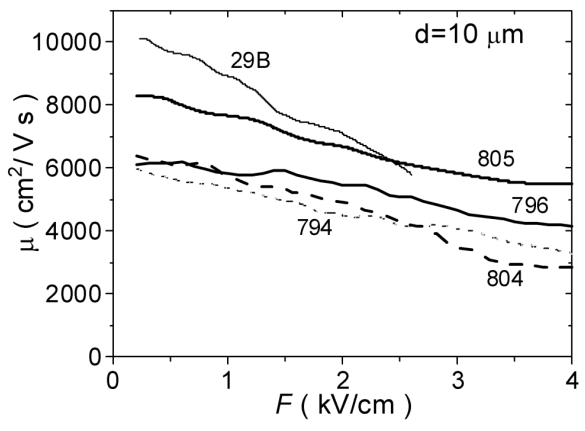

Fig. 2. Field dependence of the electron mobility in different samples. Solid and dashed lines represent the structures with the InAs inserts and without the inserts, respectively.

larger than in sample 794 and 804 without the barriers, respectively. The large mobility increase coincides with the calculated decrease of interface (IF) phonon scattering rate in the InAlAs/InGaAs structures [3, 6, 7].

A large decrease in the mobility with increase of electric fields in the range of $1-3 \mathrm{kV} / \mathrm{cm}$ is observed. This is a specific property of modulation-doped heterostructures. In these structures, electron scattering by IF phonons is the predominant scattering mechanism. The increase of electron-IF phonon scattering rate by one order, when the electron energy exceeds the IF phonon energy $(40-50 \mathrm{meV})$, is responsible for such strong mobility decrease in relatively low electric fields.

Let us note that the decrease in the mobility is responsible for the increase in the threshold electric field for the intervalley transfer of electrons, $F_{\mathrm{th}}$, which becomes larger than that in bulk InGaAs semiconductors.

The electron density in the QW channel of the modulation-doped structure is determined by the potential difference between the ionized donor layer and the QW electron channel. This potential difference depends not only on the applied voltage $V_{\mathrm{D}}$, but mostly on the preparation technology of the samples for measurements.
The potential local disturbance during preparation of ohmic contacts increases the electron density in the QW channel. In considered samples, this increase in the electron density $n_{\mathrm{s}}(0.5)$ determined from magnetoresistance measurements at low electric fields, $F=0.5 \mathrm{kV} / \mathrm{cm}$, is shown in Table.

The difference in $n_{\mathrm{s}}(0.5)$ can be explained by the difference in structures of the samples and their fabrication technique. Let us note that the value of $n_{\mathrm{s}}(0.5)$ depends on the distance between ohmic contacts (length of samples) (see Table). The large electron density change at relatively low electric fields is a distinguished property of the modulation-doped heterostructures, and is not observed in conventional field-effect transistors with a homogeneously doped QW.

\section{Negative conductivity and Gunn effect in modulation-doped structures}

A negative differential conductivity in InGaAs arises due to electron intervalley transfer at high electric fields. The threshold field for the electron intervalley transfer can be estimated from the electron energy balance equation

$$
j_{\mathrm{th}} F_{\mathrm{th}}=\Delta_{\Gamma L} / \tau_{\mathrm{E}},
$$

where $j_{\mathrm{th}}=\sigma F_{\mathrm{th}}, \sigma$ is the conductivity, $\tau_{\mathrm{E}}$ is the relaxation time of the electron energy and $\Delta_{\Gamma L}$ corresponds to the assumed electron energy, at which the electron $\Gamma-L$ intervalley transfer takes place. According to (1), the threshold field $F_{\mathrm{th}}$, and current $I_{\mathrm{th} 0}$ are equal to

$$
F_{\mathrm{th} 0}=\sqrt{\frac{\Delta_{\Gamma L}}{\tau_{\mathrm{E}} \sigma}}, \quad I_{\mathrm{th} 0}=\sqrt{\frac{\Delta_{\Gamma L} \sigma}{\tau_{\mathrm{E}}}} .
$$

Let us note that the threshold field $F_{\text {tho }}$ increases and current $I_{\text {th0 }}$ decreases with decreasing the conductivity $\sigma$ in electric fields. In bulk InGaAs, the threshold field for the intervalley transfer, $F_{\mathrm{th}}$, is around $5-7 \mathrm{kV} / \mathrm{cm}$ [8].

At magnetic field $B$ applied perpendicularly to a $\mathrm{QW}$ plane, the conductivity in samples with short length $d$ and wide width $w(d \ll w)$,

$$
\sigma(B)=\sigma_{0}\left(1+\mu^{2} B^{2}\right)^{-1} .
$$

Therefore, in the short sample, the threshold field for the intervalley transfer, $F_{\mathrm{th}}$, increases with magnetic field,

$$
F_{\text {th }}(B)=F_{\text {th } 0} \sqrt{1+\mu^{2} B^{2}},
$$

and the threshold current decreases with magnetic field,

$$
I_{\mathrm{th}}(B)=I_{\mathrm{th} 0}\left(1+\mu^{2} B^{2}\right)^{-1 / 2} \text {. }
$$

In the long samples $(d \sim w)$, the electric field is concentrated in the areas of the large magnetoresistance near the conductive contacts. Then at the same current, the electric field in this area is $\left(1+\mu^{2} B^{2}\right)$ times larger than in the remaining part of the sample. Therefore, in the long sample, the threshold mean electric field for the intervalley transfer decreases with the increase of magnetic field

$$
F_{\mathrm{th}}(B)=\frac{U_{\mathrm{th}}}{d}=\frac{F_{\mathrm{th} 0}}{\left[1+(\mu B)^{2}\right]^{1 / 2}} .
$$

Figure 3 shows the experimental dependence of the ob- 
served changes of $I(U)$ in the short sample with length $d=10 \mu \mathrm{m}$. One can see the increase in the threshold voltage with increasing magnetic field strength and the decrease in the threshold current, according to (4) and (5). Figure 4 shows the $I(U)$ dependences in the longer samples with $d=100 \mu \mathrm{m}$ and $w=100 \mu \mathrm{m}$. The threshold voltage decrease (according to (6)) is observed.

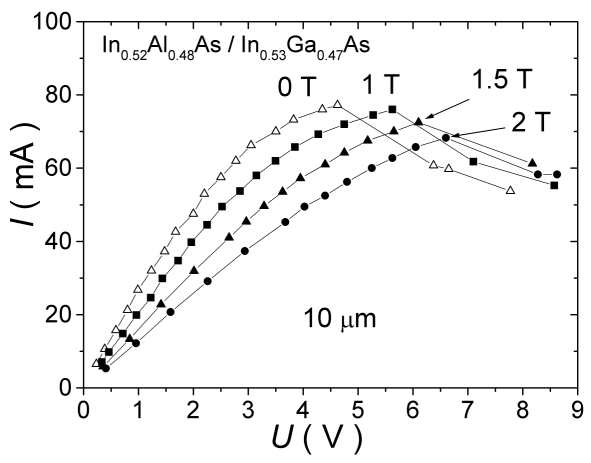

Fig. 3. Current-voltage dependences at different magnetic feld strengths $B$ from 0 to $2 \mathrm{~T}$ for sample 794 with length $d=10 \mu \mathrm{m}$.

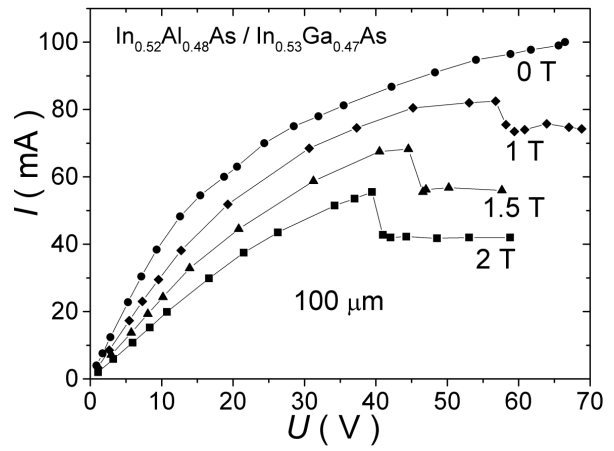

Fig. 4. Current-voltage dependences at different magnetic feld strengths $B$ from 0 to $2 \mathrm{~T}$ in sample 805 with length $d=100 \mu \mathrm{m}$. The jump in the threshold current increases with increasing $B$.

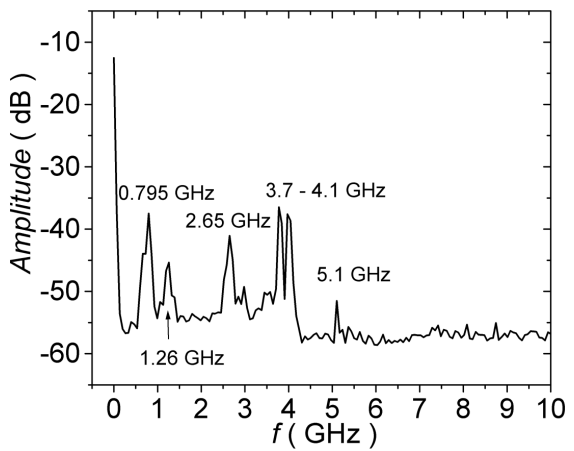

Fig. 5. Spectrum of current oscillations for sample 794 with length $d=50 \mu \mathrm{m}$ at voltage $U=22.5-23 \mathrm{~V}$ and $B=0$.
The $I(U)$ dependence demonstrates the negative differential conductivity at $U>U_{\mathrm{th}}$. The great perturbation of potential along the QW channel and therefore the change in the electron density is created when the negative differential conductivity in QW takes place. At large magnetic fields, $B>1 \mathrm{~T}$, the sharp jump of the current at $U \geq U_{\text {th }}$ and the following saturation of the current is observed (see Fig. 4). As a result, the current oscillation takes place in a narrow range of applied voltages and magnetic field values. The spectrum of oscillations in the gigahertz frequency range measured using Agilent Spectrum Analyzer E 4407B is shown in Fig. 5. Let us note that the oscillations are observed in a very narrow interval of the voltage applied to the sample near to the threshold voltage $U_{\mathrm{th}}=22.5 \mathrm{~V}$. The frequencies of oscillations coincide with the drift time of electrons along the samples: $t \approx 10^{-10} \mathrm{~s}$ at $U_{\mathrm{th}} \approx 22.5 \mathrm{~V}$.

\section{Conclusions}

1. The enhancement of the electron mobility by inserting an InAs phonon wall into the $\operatorname{In}_{0.53} \mathrm{Ga}_{0.47} \mathrm{As} /$ $\mathrm{In}_{0.52} \mathrm{Al}_{0.48} \mathrm{As}$ quantum well, as well as increasing the InAs content in the modulation-doped $\operatorname{In}_{0.8} \mathrm{Ga}_{0.2} \mathrm{As} /$ $\mathrm{In}_{0.7} \mathrm{Al}_{0.3}$ As heterostructure is confirmed experimentally.

2 . In the modulation-doped heterostructure, the decrease of the mobility and change in electron density takes place at low electric fields $(1-3 \mathrm{kV} / \mathrm{cm})$. This is a distinguished property of the modulation-doped heterostructures.

3. The magnetic field decreases the threshold current for the intervalley scattering. Current oscillations in the $\mathrm{GHz}$ range are observed in a narrow range of applied voltages and magnetic field values.

\section{References}

[1] K. Onda, A. Fujihara, A. Vakejima, E. Mizuki, T. Nakayama, H. Miyamoto, Y. Ando, M. Kanamori, IEEE Electron Dev. Lett. 19, 300 (1998).

[2] H. Zhao, Y.-T. Chen, J.H. Yum, Y. Wang, F. Zhou, F. Xue, J.C. Lee, Appl. Phys. Lett. 96, 102101 (2010).

[3] V.G. Mokerov, I.S. Vasil'evskii, G.B. Galiev, J. Požela, K. Požela, A. Sužiedèlis, V. Jucienè, Č. Paškevič, Fiz. Tekh. Poluprovodn. 43, 478 (2009) [Semiconductors 43, 458 (2009)].

[4] N. Dyakonova, A. El Fatimy, J. Lusakowski, W. Knap, Appl. Phys. Lett. 88, 141906 (2006).

[5] W.J. Stillman, M.S. Shur, J. Nanoelectron. Optoelectron. 2, 209 (2007).

[6] I.S. Vasil'evskii, G.B. Galiev, Yu.A. Matveev, E.A. Klimov, J. Požela, K. Požela, A. Sužiedèlis, C. Paškevič, V. Jucienè, Fiz. Tekh. Poluprovodn. 44, 928 (2009) [Semiconductors 44, 898 (2010)].

[7] J. Požela, K. Požela, V. Jucienè, A. Shkolnik, Semicond. Sci. Technol. 25, 014025 (2011).

[8] M. Inoue, K. Ashida, T. Sugino, J. Shirafuji, Y. Inuishi, Jpn. J. Appl. Phys. 12, 932 (1973). 